\title{
A atuação da(o) enfermeira(o) na classificação de risco em obstetrícia: uma revisão integrativa
}

RESUMO | Objetivo: apresentar as evidências científicas sobre a atuação da(o) enfermeira(o) na classificação de risco em obstetrícia. Método: trata-se de um estudo qualitativo, do tipo revisão integrativa, realizado nas bases de dados SciELO e PubMed, na série temporal de 2011 a 2021, no idioma português, com os descritores e operadores boleanos. Resultados: Identificou-se um conjunto de 105 publicações sobre a temática no SciELO e 86 na PubMed. Do total de 191 publicações identificadas, segundo a combinação dos descritores e acréscimos de booleanos, 4 foram consideradas para análise, que demonstravam $\mathrm{a}(\mathrm{o})$ enfermeira(o) como principal profissional na classificação de risco às gestantes devido à sua assistência eficaz, ágil e resolutiva, reduzindo os riscos à saúde do binômio e impactando sobre a mortalidade maternoinfantil. Conclusão: percebe-se que a utilização da Classificação de Risco às gestantes e à sua realização pela Enfermagem, proporciona melhorias no serviço, promovendo eficácia no atendimento as urgências e emergências obstétricas. Palavras-chaves: Risco; Obstetrícia; Saúde da Mulher; Enfermagem.

\begin{abstract}
Objective: to present the scientific evidence on the role of the nurse in the classification of risk in obstetrics. Method: this is a qualitative study, integrative review type, carried out in the SciELO and PubMed databases, in the time series from 2011 to 2021, in Portuguese, with Boolean descriptors and operators. Results: A set of 105 publications on the subject in SciELO and 86 in PubMed were identified. Of the 191 publications identified, according to the combination of descriptors and additions of Booleans, 4 were considered for analysis, which showed the nurse as the main professional in the risk classification of pregnant women due to their effective, agile and resolution, reducing the health risks of the binomial and impacting on maternal and child mortality. Conclusion: it is noticed that the use of the Risk Classification for pregnant women and its performance by Nursing, provides improvements in the service, promoting effectiveness in attending obstetric urgencies and emergencies.
\end{abstract}

Keywords: Risk; Obstetrics; Women's Health; Nursing.

RESUMEN | Objetivo: presentar la evidencia científica sobre el rol de la enfermera en la clasificación de riesgo en obstetricia. Método: se trata de un estudio cualitativo, de tipo revisión integradora, realizado en las bases de datos SciELO y PubMed, en la serie temporal de 2011 a 2021, en portugués, con descriptores y operadores booleanos. Resultados: Se identificó un conjunto de 105 publicaciones sobre el tema en SciELO y 86 en PubMed. De las 191 publicaciones identificadas, de acuerdo a la combinación de descriptores y adiciones de booleanos, se consideraron para análisis 4, que mostraron al enfermero como el principal profesional en la clasificación de riesgo de la gestante por su eficacia, agilidad y resolución, reduciendo la salud. riesgos del binomio y que impactan en la mortalidad materna e infantil. Conclusión: se advierte que el uso de la Clasificación de Riesgo para gestantes y su desempeño por parte de Enfermería, brinda mejoras en el servicio, promoviendo la efectividad en la atención de urgencias y emergencias obstétricas.

Palabras claves: Riesgo; Obstetricia; La salud de la mujer; Enfermería.

\section{Michelle Araújo Moreira}

Enfermeira. Professora Titular do Curso de Enfermagem da Universidade Estadual de Santa Cruz (UESC), Pós-doutora em Enfermagem, Doutora em Enfermagem, Mestre em Enfermagem, Especialista em Saúde Pública, Especialista em Enfermagem Obstétrica, Especialista em Cuidado Pré-natal. ORCID: 0000-0002-6998-8665

\section{Miriam Santos Carvalho}

Graduanda de Enfermagem, Universidade Estadual de Santa Cruz (UESC).

ORCID: 0000-0002-8490-6971

\section{José Carlos de Araújo Junior}

Enfermeiro. Mestre em Enfermagem, Especialista em Enfermagem Obstétrica. Professor Assistente do Curso de Enfermagem da Universidade Estadual de Santa Cruz (UESC). ORCID: 0000-0001-7913-5108

\section{Polliana Santos Ribeiro}

Enfermeira. Residente em Saúde da Família, Universidade Estadual de Santa Cruz (UESC). ORCID: 0000-0002-8041-0231

\section{Carla Daiane Costa Dutra}

Mestre em Desenvolvimento Regional e Meio Ambiente, Doutoranda em Ciências, Especialista em Gerontologia, Especialista em Pedagogias Diferenciadas e Especialista em Gestão da Atenção Básica, Enfermeira. Professora Assistente do Curso de Enfermagem da Universidade Estadual de Santa Cruz (UESC).

ORCID: 0000-0003-0866-1519

\section{Patrícia Figueiredo Marques}

Professora Adjunta do Curso de Enfermagem da Universidade Federal do Recôncavo da Bahia (UFRB), Mestre em Enfermagem, Doutora em Enfermagem,
Especialista em Metodologia do Ensino Superior, Enfermeira.

ORCID: 0000-0002.0242-5024

Recebido em: 04/06/2021

Aprovado em: 11/06/2021

INTRODUÇÃO

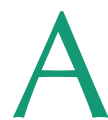
gestação é um processo fisiológico demarcado por alterações físicas e emocionais ocorridas no organismo da mulher. Por vezes, o desenvolvimento desse processo culmina em modificações que desfavorecem a saúde da gestante e/ou feto, sendo considerada uma gestação de risco. Sabe-se que, a ausência de acompanhamento pré-natal e da avaliação de risco incidem em uma maior probabilidade de agravos 
e complicações que ameaçam a vida do binômio e aumentam o índice de morte materna e fetal. ${ }^{(1)}$

No Brasil, a morte materna configura-se como um grave problema de saúde pública. Para tanto, em 2011, o Ministério da Saúde instituiu a Rede Cegonha, como estratégia para ampliar a excelência na atenção ao pré-natal, parto e puerpério e desenvolvimento da criança até os dois anos de idade, através da garantia de acolhimento e classificação de risco. ${ }^{(2)}$

Por sua vez, o acolhimento garante a integralidade do cuidado a todos os usuários que adentrem os serviços de saúde, e envolve o quadro de profissionais dessas instituições. Todavia, os problemas de superlotação dos serviços, os altos índices de mortalidade materna e complicações no parto e puerpério não podem ser sanados apenas com o acolhimento, para isso, o acolhimento precisa ser realizado com a classificação de risco do binômio. Sendo assim, o Acolhimento e Classificação de Risco torna-se um dispositivo de organização dos fluxos, com base em critérios que visam priorizar o atendimento as gestantes que apresentam sinais e sintomas de maior gravidade e ordenar toda a demanda. Ele se inicia no momento da chegada da muIher, com a identificação da situação/queixa ou evento apresentado por ela. ${ }^{(3)}$

Para garantir que o acesso seja integral e de qualidade, que ocorra com agilidade e de maneira humanizada, são utilizados protocolos que sistematizam o atendimento. O Sistema de Triagem de Manchester apresenta boa confiabilidade, pois identifica precocemente a gestante que precisa de intervenção ou cuidado precoce. Este sistema determina a classificação do risco a partir de cores: vermelho (emergência); laranja (muito urgente); amarelo (urgente); verde (pouco urgente) e azul (não urgente) e o tempo para cada atendimento. ${ }^{(4)}$

Ressalta-se ainda que, de acordo com o Conselho Federal de Enfermagem descrita na Resolução $n^{\circ}$ 661/21, no âmbito da equipe de enfermagem, compete privativamente $\mathrm{a}(\mathrm{o})$ enfermeira(o) a realiza-
No Brasil, a morte materna configurase como um grave problema de saúde pública. Para tanto, em 2011, o Ministério da Saúde instituiu a Rede Cegonha, como estratégia para ampliar a excelência na atenção ao pré-natal, parto e puerpério e desenvolvimento da criança até os dois anos de idade, através da garantia de acolhimento e classificação de risco. ção da Classificação de Risco e priorização da assistência. ${ }^{(5)}$

Diante do exposto, emergiram os seguintes questionamentos: Quais as evidências científicas encontradas na literatura sobre a atuação da(o) enfermeira(o) na classificação de risco em obstetrícia?

Desse modo, obteve-se como objetivo geral: Apresentar as evidências científicas sobre a atuação da(o) enfermeira(o) na classificação de risco em obstetrícia. Para tanto, justifica-se a necessidade da pesquisa em virtude da baixa quantidade de produções científicas sobre a temática, algo percebido em um levantamento prévio nas bases de dados SciELO e PubMed nos últimos 5 anos.

Por fim, a relevância científica e social que versou a pesquisa, possibilita a atenção dos profissionais de saúde quanto a importância da Classificação de Risco em Obstetrícia como ferramenta estratégica de avaliação de riscos e atendimentos prioritários nos serviços de saúde com vistas à redução da morbimortalidade materna e neonatal, dispondo a(o) enfermeira(o) como elemento fundamental nesse processo.

\section{MÉTODO}

Trata-se de uma pesquisa descritiva, exploratória, de abordagem qualitativa, do tipo revisão integrativa da literatura, que possibilita a geração de novos conhecimentos sobre um determinado tema, bem como a revisão de tópicos emergentes. ${ }^{(6)}$

Para a construção metodológica da revisão integrativa, utilizou-se o esquema dividido em cinco etapas: elaboração da questão de pesquisa, construção do objetivo, delimitação do tópico de interesse da revisão, estabelecimento das estratégias de busca, critérios de exclusão e inclusão, realização da pesquisa nas bases de dados, avaliação e análise dos dados, e apresentação dos resultados. ${ }^{(7-8)}$

Ao ser estabelecida a primeira etapa, formula-se a questão de pesquisa através de critérios, com o objetivo de definir o 
problema, os descritores e as estratégias de buscas nas fontes de dados, conforme o anagrama PICo: P- população alvo; IFenômeno de interesse; Co- Contexto(9), correspondendo a: P - Enfermeiras(os); I Atuação da(o) enfermeira(o) na classificação de risco; Co - Serviços de saúde obstétricos. Ao final, tem-se a seguinte questão: Como se dá a atuação da(o) enfermeira(o) na classificação de risco em obstetrícia?

Levantada a questão de pesquisa, para segunda etapa do processo foram estabelecidos os critérios de inclusão e exclusão dos estudos para delimitação das buscas nas bases de dados. As estratégias de busca foram elaboradas conforme as especificidades de cada base de dados, foram escolhidos descritores a partir dos Descritores em Ciências da Saúde (DeCS) acrescidos do operador booleano "AND". Para a seleção dos estudos, os critérios de inclusão foram: artigos científicos, publicados em português, gratuitos, na série temporal de 2011 a 2021 que abordassem a atuação da(o) enfermeira(o) na Classificação de Risco em obstetrícia. Como critérios de exclusão foram estabelecidos: teses, dissertações, capítulos de livros, textos não científicos, editoriais, anais de eventos e resenhas de livros.

Assim, a busca dos acervos a serem utilizados partiram das bases de dados: SciELO e PubMed. No SciELO, foram utilizados os seguintes descritores: ("Enfermagem" and "Acolhimento") com 48 artigos encontrados e 2 selecionados; ("Classificação de risco" and "Enfermagem") com 16 artigos encontrados e 1 selecionado; e ("Enfermagem" and "Obstetrícia") com 40 artigos encontrados e nenhum selecionado. Na PubMed: "Enfermagem" and "Acolhimento") com 12 artigos encontrados e nenhum selecionado; ("Classificação de risco" and "Enfermagem") encontrados 7 artigos e nenhum selecionado; e ("Enfermagem" and "Obstetrícia") 67 artigos encontrados e 1 selecionado.

Identificou-se um conjunto de 105 publicações sobre a temática no SciELO e 86 na PubMed. Do total de 191 publicações identificadas, segundo a combinação dos descritores e acréscimos de booleanos, 4 foram consideradas para análise.

A seleção dos estudos foi feita através da aplicação do fluxograma Preferred Reporting Items for Systematic Reviews and Meta-Analyses (PRISMA), na sequência a seguir ${ }^{(10)}$ : número de registros identificados nas bases de dados, acervos excluídos, seleção após aplicação do operador
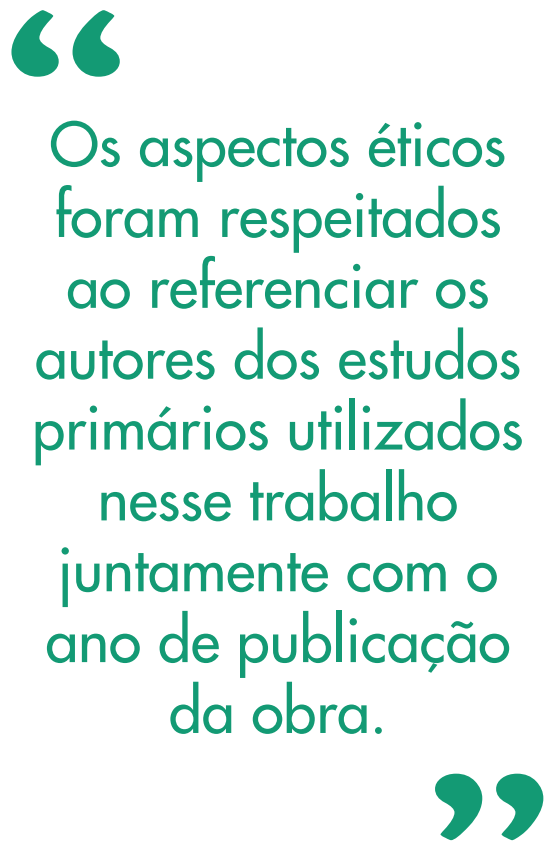

booleano aos descritores, número de artigos excluídos após leitura completa do texto, número de artigos elegíveis após a leitura completa dos textos, artigos incluídos na revisão integrativa.

$\mathrm{Na}$ terceira etapa ocorreu as definições das informações a serem extraídas dos estudos selecionados com formação de um banco de dados de fácil acesso e manejo. ${ }^{(11)}$ A quarta e quinta etapas compreenderam a análise dos dados e a interpretação dos resultados. ${ }^{(7)}$

Os aspectos éticos foram respeitados ao referenciar os autores dos estudos primários utilizados nesse trabalho juntamente com o ano de publicação da obra.

\section{APRESENTAÇÃO DOS RESULTADOS E DISCUSSÃO}

Para apresentação dos estudos incluídos na revisão integrativa utilizou-se o fluxograma PRISMA, conforme ilustrado na Figura 1:

1.067 artigos dispostos nas bases de dados, sendo 574 da PubMed e 493 da SciELO, após utilização dos critérios de seleção acrescidos do operador booleano "AND", foram encontrados 191 artigos, 86 da PubMed e 105 da SciELO. Após a leitura completa dos textos, visando uma aborda-

Figura 1 - Fluxograma informatio das etapas da revisão integrativa ancorado no PRISMA, Itabuna - BA, Brasil, 2021.

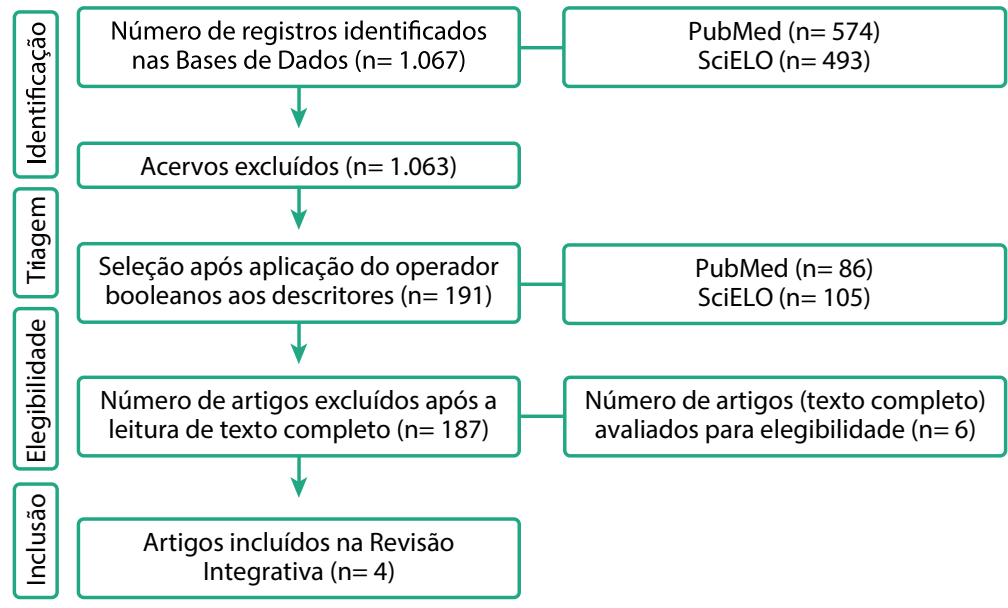

Fonte: Elaborado pelos autores, 2021. 
gem sobre a atuação da(o) enfermeira(o) na classificação de risco em obstetrícia, foram selecionados 4 artigos sobre o objeto, sendo 3 da base de dados SciELO e 1 da Pubmed.

$\mathrm{Na}$ distribuição dos artigos científicos, que formaram o corpo do estudo, destacam-se o título, o periódico, o ano de publicação, os autores e as considerações finais a respeito da atuação da(o) enfermeira(o) no processo de classificação de risco em obstetrícia, os quais estão dispostos no Quadro 1 a seguir:

Os estudos brasileiros utilizaram metodologias distintas, mas abordavam a classificação de risco em obstetrícia como uma ferramenta de apoio à decisão para identificação imediata da gravidade da gestante, orientados pela aplicação do protocolo de Manchester.

Destaca-se que, a(o) enfermeira(o) atua como comunicador às gestantes e seus fiIhos, contribuindo para uma assistência adequada como princípio fundamental da Rede Cegonha ${ }^{(16)}$ e da Política Nacional de Humanização da Atenção e Gestão do Sistema Único de Saúde. ${ }^{(17)}$ Evidenciou-se que, a(o) enfermeira(o) obteve participação ativa na classificação de risco, demons- trando a importância da sua atuação para salvaguardar a vida das gestantes e de seus filhos em virtude da sua eficiência, agilidade e capacidade de resolutividade. Nesse sentido, a(o) enfermeira(o) contribui para reduzir os riscos à saúde do binômio, impactando nos indicadores de morbimortalidade materna e infantil nos mais variados níveis de complexidade. ${ }^{(13)}$

Ressalta-se ainda que, o processo de trabalho de classificar os riscos à gestante, deve envolver todos os profissionais de saúde, além da(o) enfermeira(o), por meio de um cuidado horizontal, com satisfação da(s) usuária(s). ${ }^{(14)}$

Ademais, as evidências científicas demonstram que a classificação de risco quando executada de forma adequada contribui para a diminuição dos índices de abortamento e de morte materna/infantil em virtude da tomada rápida das decisões com chances reais de oportunizar uma vivência do ciclo gravídico-puerperal com preservação da saúde do binômio. ${ }^{15,12)}$

Desse modo, entende-se que a(o) enfermeira(o) consegue classificar o risco em obstetrícia com maestria em virtude das características inerentes à sua profis- são, tais como: olhar social, emocional, biológico e técnico sobre as problemáticas principalmente quando consideramos suas competências e habilidades, atuando sobre situações de alto risco obstétrico. ${ }^{(18)}$

Para tanto, a(o) enfermeira(o) executa este processo em duas fases: a implantação e a operacionalização. $\mathrm{Na}$ implantação, a(o) enfermeira(o) planeja recursos físicos, materiais e humanos, capacita profissionais e usuárias, organiza o processo de trabalho com controle da demanda, triando o que pode ser entendido como emergência obstétrica e o que pode ser atendido em outros serviços de menor complexidade. Na operacionalização da classificação de risco utiliza conhecimento atual e comunicação apropriada da Enfermagem bem como de vários outros profissionais da saúde para o cuidado às gestantes, parturientes, puérperas e suas famílias. ${ }^{(19)}$

Por fim, nota-se que a classificação de risco em obstetrícia realizada pela(o) enfermeira(o) melhora o acesso das usuárias, aumentando a relação de confiabilidade e permitindo que a humanização seja condição sine qua non para uma assistência

Quadro 1 - Distribuição dos artigos selecionados segundo título, base de dados, ano, autoria e considerações. Itabuna-BA, Brasil, 2021.

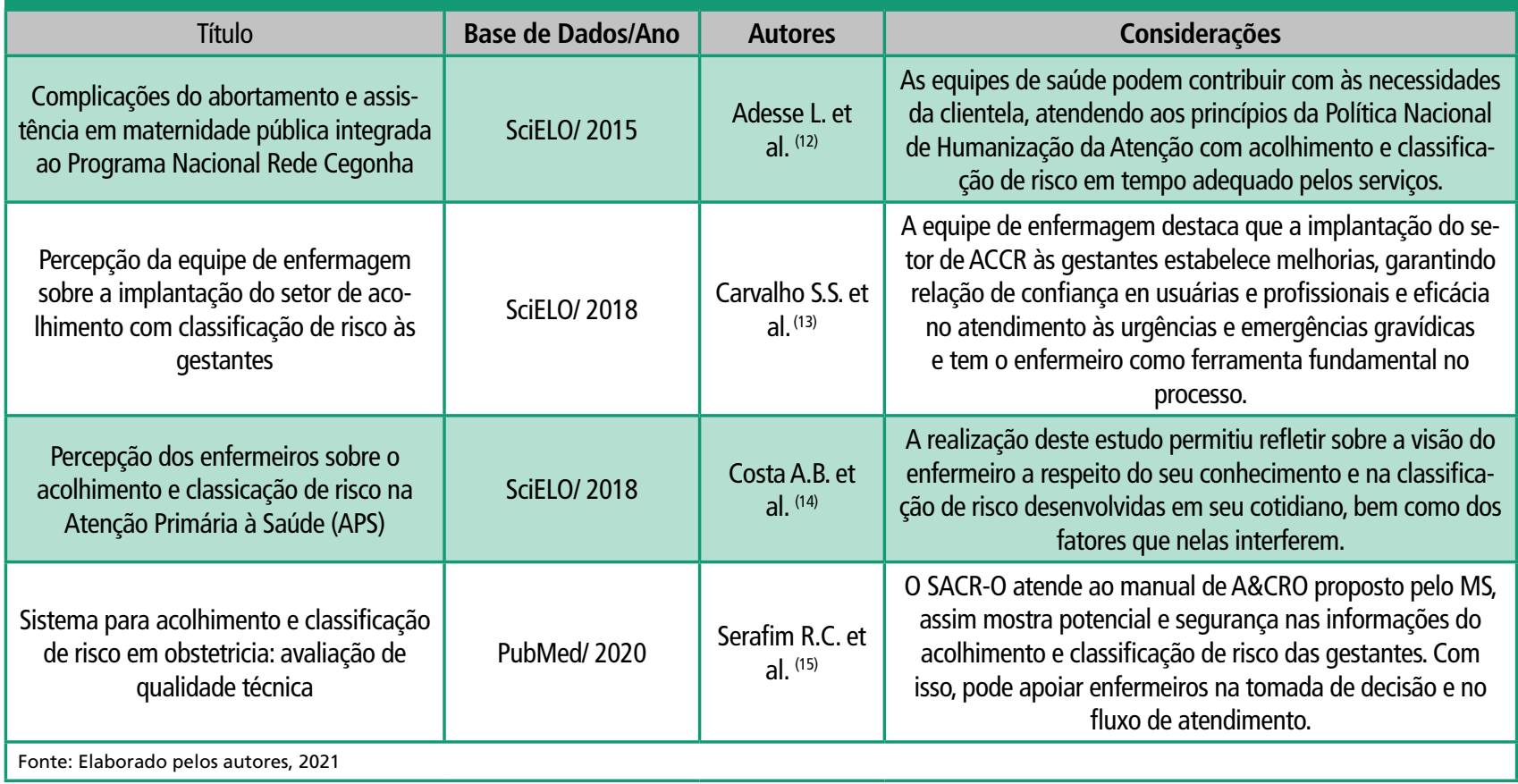


adequada. ${ }^{(20)}$ Além disso, este processo permite a utilização das tecnologias duras, leves-duras e leves durante o trabalho da Enfermagem com vistas a garantir escuta, orientação, agregação da equipe e usuária e a valorização de saberes. ${ }^{(18)}$

\section{CONCLUSÃO}

Percebe-se que, a utilização da
Classificação de Risco às gestantes e à sua realização pela(o) enfermeira(o), proporciona melhorias nos serviços de saúde, auxílio na tomada de decisão, promovendo um atendimento eficaz, sendo as gestantes classificadas em tempo oportuno para recebimento de uma assistência qualificada, humanizada e resolutiva, baseada em evidências científicas. Recomenda-se que sejam realizadas ações para capacitação de profissionais nos serviços de saúde, discentes da graduação e pós-graduação na área de enfermagem, com destaque para a classificação de risco na obstetrícia. Salienta-se a importância de estratégias por parte de gestores para implantação e implementação da proposta com contínua avaliação para meIhorar sua eficiência.

\section{Referências}

1. Correia RA, Rodrigues ARM, Araújo PF, Monte AS. Análise do acolhimento com classificação de risco em uma maternidade pública terciária de Fortaleza. Enferm. foco. 2019; 10(1): 105-10. Disponível em: <http://revista.cofen.gov. br/index.php/enfermagem/article/view/1448>. DOI: https://doi.org/10.21675/ 2357-707X.2019.v10.n1.1448.

2. Brasil. Ministério da Saúde. Manual de acolhimento e classificação de risco em obstetrícia. Brasília: Ministério da Saúde, 2018. Disponível em: https:// portaldeboaspraticas.iff.fiocruz.br/wp-content/uploads/2018/10/Manual_obstetr\%C3\%ADcia-final-1.pdf.

3. Brasil. Ministério da Saúde. Atenção as mulheres: acolhimento e classificação de risco em serviços de obstetrícia. Brasília: Ministério da Saúde. 2018. Disponivel em: <http://portaldeboaspraticas.iff.fiocruz.br/atencao-mulher/ acolhimento-e-classificacao-de-risco-em-obstetricial>.

4. Pereira ALF, Lima AEF. Acolhimento com classificação de risco em uma maternidade pública. Rev enferm UFPE on line. 2014; 8(supl. 1): 2309-15. Disponível em: file:///C:/Users/User/Downloads/9920-18965-1-PB.pdf. DOI: 10.5205/ reuol.5927-50900-1-SM.0807supl201416.

5. Conselho Federal de Enfermagem. Resolução COFEN nº61/2021. Atualiza e normatiza, no âmbito do Sistema Cofen/Conselhos Regionais de enfermagem, a participação da equipe de enfermagem na atividade de Classificação de Risco. Brasília: Conselho Federal de Enfermagem. 2021. Disponível em: http:// www.cofen.gov.br/resolucao-cofen-no-661-2021_85839.html.

6. Torraco RJ. Writing integrative literature reviews: Using the past and present to explore the future. Hum. resour. development rev. 2016; 15(4): 404-28. Disponível em: <https://journals.sagepub.com/doi/ abs/10.1177/1534484316671606?journalCode=hrda>. DOl: https://doi. org/10.1177/1534484316671606.

7. Whittemore R, Knafl K. The integrative review: update methodology. J. adv. nurs. 2005; 52(5): 546-53. DOI: 10.1111/j.1365-2648.2005.03621.x.

8. Fossati EC, Mozzato AR, Moreto CF. 0 uso da revisão integrativa na administração: um método possível?. Rev. eletrônica cient do CRA-PR. 2019; 6(1): 55-72. Disponivel em: <http://recc.cra-pr.org.br/index.php/recc/article/viewFile/169/122>

9. Donato H, Donato M. Etapas da condução de uma revisão sistemática. Acta med. port. 2019; 32(3): 227-35. Disponivel em:

$<$ https://www.researchgate.net/publication/332084935_Etapas_na_Conducao_de_uma_Revisao_Sistematica>. DOI: https://doi.org/10.20344/amp.11923. 10. Moher D, Liberati A, Tetzlaff J, Altman DG. The PRISMA Group. Preferred Reporting Items for Systematic Reviews and Meta-Analyses: The PRISMA Statement. Epidemiol. Serv. Saúde (Online). 2015; 2(24): e1000097. Disponível em: www.prisma-statement.org. DOI: https://doi.org/10.1371/journal. pmed.1000097.

11. Botelho LLR, Cunha CCA, Macedo M. 0 método da revisão integrativa nos estudos organizacionais. Gestão \& Sociedade. 2011; 5(11): 121-36. Disponível em: <https://www.gestaoesociedade.org/gestaoesociedade/article/ view/1220/906>. DOI: https://doi.org/10.21171/ges.v5i11.1220.

12. Adesse L, Silva KS, Bonan C, Fonseca VM. Complicações do abortamento e assistência em maternidade pública integrada ao Programa Nacional Rede Cegonha. Saúde debate. 2015; 39(106): 694-706. Disponível em: https://www. scielo.br/pdf/sdeb/v39n106/0103-1104-sdeb-39-106 00694.pdf. DOI: https:// doi.org/10.1590/0103-1104201510600030011.

13. Carvalho SS, Oliveira BR, Nascimento CSO, Gois CTS, Pinto IO. Percepção da equipe de enfermagem sobre a implantação do setor de acolhimento com classificação de risco às gestantes. Rev. Bras. Saúde Mater. Infant (Online). 2018; 18(2): 301-7. Disponível em: <https://www.scielo.br/j/rbsmi/a/ wpKrBYRpdwthfZrDDVjSDTR/?lang=pt>. DOl: https://doi.org/10.1590/180693042018000200004.

14. Costa AB, Gouvea PB, Rangel RCT, Scnheider P, Alves TF, Acosta AS. Percepção dos enfermeiros sobre o acolhimento e classificação de risco na Atenção Primária à Saúde (APS). Enferm. actual Costa Rica (Online). 2018;1(35): 103-15. Disponivel em: <https://search.scielo.org/?lang=pt\&count=15\&from $=0$ \&output $=$ site $\&$ sort $=\&$ format $=$ summary $\& \mathrm{fb}=\&$ page $=1 \& \mathrm{q}=$ Percep $\%$ $\mathrm{C} 3 \% \mathrm{~A} 7 \% \mathrm{C} 3 \% \mathrm{~A} 30+$ dos+enfermeiros+sobre+0+acolhimento+e+classifica $\% \mathrm{C} 3 \% \mathrm{~A} 7 \% \mathrm{C} 3 \% \mathrm{~A} 30+$ de + risco+na+Aten $\% \mathrm{C} 3 \% \mathrm{~A} 7 \% \mathrm{C} 3 \% \mathrm{~A} 30+$ Prim $\%$ C3\%A1 ria+\%C3\%A0+Sa\%C3\%BAde+\%28APS\%29>. DOl: http://dx.doi. org/10.15517/revenf.v0i35.32113.

15. Serafim RC, Temer MJ, Parada CMGL, Peres HHC, Serafim CTR, Jensen R. Sistema de acolhimento e classificação de risco em obstetrícia: uma avaliação da qualidade técnica. Rev. Latinoam. Enferm (Online). 2020; 28(e3330): 1-8. Disponivel em: https://pubmed.ncbi.nlm.nih.gov/32696923/. https://doi. org/10.1590/1518-8345.3327.3330.

16. Rodrigues AFM, Candido CL, Campos GKP, Barcellos JES, Rodrigues LA, Seidel TS. Pré-natal na atenção primária, adequação das consultas e avaliação da assistência às gestantes: revisão integrativa. Revista Nursing. 2021; 24(275): 5484-9. Disponível em: http://revistas.mpmcomunicacao.com.br/index.php/ revistanursing/article/view/1471/1674. DOI: https://doi.org/10.36489/nursing 2021v24i275p5484-5495.

17. Brasil. Ministério da Saúde. Acolhimento nas práticas de produção de saúde. Brasília: Ministério da Saúde. 2010. Disponível em: <https://bvsms.saude. gov.br/bvs/publicacoes/acolhimento_praticas_producao_saude.pdf. 18. Rates HF, Alves M, Cavalcante RB. 0 processo de trabalho do enfermeiro no acolhimento com classificação de risco. Rev REME. 2016; 20(e969): 1-6. Disponivel em: file:///C:/Users/User/Downloads/0\%20PROCESSO $\% 20$ DE\%20TRABALHO\%20D0\%20ENFERMEIRO\%20(1).pdf. DOI: 10.5935/14152762.20160039.

19. Oliveira JLC, Souza VS, Inoue KC, Costa MAR, Camillo NRS, Matsuda LM. Atuação do enfermeiro no acolhimento com classificação de risco: um estudo de metassíntese. Cienc Cuid Saude. 2016; 15(2): 374-82. Disponível em: file:///C:/Users/User/Downloads/ATUA\%C3\%87\%C3\%830\%20D0\%20ENFERMEIRO \%20NO\%20ACOLHIMENTO\%20CR\%20-\%20metass\%C3\%ADntese. pdf. DOI: 10.4025/cienccuidsaude.v15i2.28573.

20. Neto OC, Andrade GKS, Karpiuckc LB, Ganassind AR. A Atuação do Enfermeiro no Sistema de Acolhimento e Classificação de Risco nos Serviços de Saúde. J Health Sci. 2018; 20(4): 295-302. Disponivel em: file:///C:/Users/User/ Downloads/A\%20ATUA\%C3\%87\%C3\%830\%20D0\%20ENFERMEIRO $\% 20$ NO $\% 20$ SISTEMA $\% 20$ DE $\% 20$ ACOLHIMENTO $\% 20$ E $\% 20$ CR $\% 20$ NOS $\% 20$ SERVI\%C3\%870S\%20DE\%20SA\%C3\%9ADE.pdf. DOI: http://dx.doi.org/10.17921/2447-8938.2018v20n4p295-302. 